\title{
A Dual Band Elliptical DRA
}

\author{
Jian-Juang Chen*, Yi-Cheng Lin and Ruey-Beei Wu
}

Department of Electrical Engineering and Graduate Institute of

Communication Engineering,

National Taiwan University, Taipei, Taiwan, 10617, ROC

E-mail: r91060@ew.ee.ntw.edu.tw, yclin@ew.ee.ntu.edu.tw

Abstract: This paper presents a dual band frequency antenna by using an elliptical dielectric resonator antenna. We describe an elliptical dielectric resonator antenna on a ground plane. Dual bands are obtained by utilizing two coaxial feeds on two sides of the elliptical DRA. A simulation result of the return loss and the radiation characteristic of the elliptical DRA is included.

\section{Introduction}

In recent years dielectric resonator antennas (DRAs) have been widely studied by many researchers [1-2]. The features and flexibility of DRAs antennas are their high radiation efficiency, large bandwidth, simple coupling, low cost, small size and so on. In this paper, we present a dual band antenna by using a single elliptical DRA. The elliptical DRA is fed by two coaxial cables, and the dual frequency is designed. The size, return loss and radiation patterns of the elliptical DRA are simulated shown in the paper. All simulation results in this paper are based on the full wave analysis of finite element methods through the commercial software HFSS [3].

\section{Antenna Structures}

In order to generate the dominant modes(HEM $11 \delta$ ) of cylindrical DRAs [4], we choose two ports to excite different frequency, and by using two ports to receive different frequency signal, we can omit the diplexer in the circuit. Figure 1 and Figure 2 show the geometry of the elliptical DRA on the ground plane. The relative permittivity $\varepsilon_{\mathrm{r}}$ of the elliptical DR is 37 and the ground plane is $50.8 \mathrm{~mm} \times 50.8 \mathrm{~mm}$. The long radius (R) of the elliptical antenna is $15 \mathrm{~mm}$ for exciting the lower frequency while the short radius ( $r$ ) of the elliptical DRA is $4.6 \mathrm{~mm}$ for exciting the upper frequency. The height $(\mathrm{H})$ of the DR is $9 \mathrm{~mm}$ and the DR is fed by two $50 \Omega$ coaxial cables with the same phase. Probe of Port 1 is bent at the top corner of DRA and extended $4 \mathrm{~mm}$ inward to match the $50 \Omega$ impedance. While the probe of of Port 2 is $5 \mathrm{~mm}$, less than the elliptical DRA height, for impedance matching.

\section{Results}

The return loss of the elliptical DRA fed by two coaxial cables is show in Figure 3. The long axis is resonant at $2.44 \mathrm{GHz}$, and the short axis is resonant at $3.36 \mathrm{GHz}$. The pattern of an elliptical DRA fed by Port 1 to excite lower resonant frequency is equivalent to that of a magnetic dipole lying along $x$ axis and its $H$-plane (xz-plane) and E-plane (yz-plane) are respectively shown in Figure 4 and Figure 5. The max directivity of the lower resonant frequency is $4.9 \mathrm{dBi}$. In this case, the mode of the 
lower frequency is HEM $11 \delta$. On the other hand, the pattern of an elliptical DRA fed by Port 2 to excite upper resonant frequency is equivalent to that of a magnetic dipole lying along y axis and its E-plane(xz-plane) and H-plane(yz-plane) are respectively shown in Figure 6 and Figure 7. The max directivity of the upper resonant frequency is $6.1 \mathrm{dBi}$. In this case, the mode of the upper frequency is also HEM11 $\delta$.

\section{Conclusions}

This paper presents a simple structure with dual bands and the performance of the elliptical DRA. The elliptical DRA size is compact and easy to manufacture. Moreover, impedance match is also solved by extending or shortening the probe of each port. According to the applications, users can choose suitable elliptical radius to generate desired frequency and tune the length of each probe to match well.

\section{Acknowledgment}

This study is supported in part of NSC, Taiwan.

\section{References}

[1] R. k. Mongia and A. Ittipiboon, "Theoretical and Experimental Investigations on Rectangular Dielectric Resonator Antennas," IEEE Trans. Antenna Propagat., vol. 45, no. 9, pp. 1348-1356, September 1997

[2] Stuart A. Long, Mark W. McAllister, and Liang C. Shen, "The Resonant Cylindrica Dielectric Cavity Antenna," IEEE Ttrans. Antenna Propagat., vol. 31, no. 3, pp. 406412, May 1983

[3] ANSOFT-HFSS 8.0

[4] R. k. Mongia and P. Bhartia, "Dielectric Resonator Antennas-A Review and General Design Relations foe Resonant Frequency and Bandwidth," International Journal of Microwave and Millimeter-Wave Computer-Aided Engineering, v 4, n 3, Jul, 1994, p 230-247 


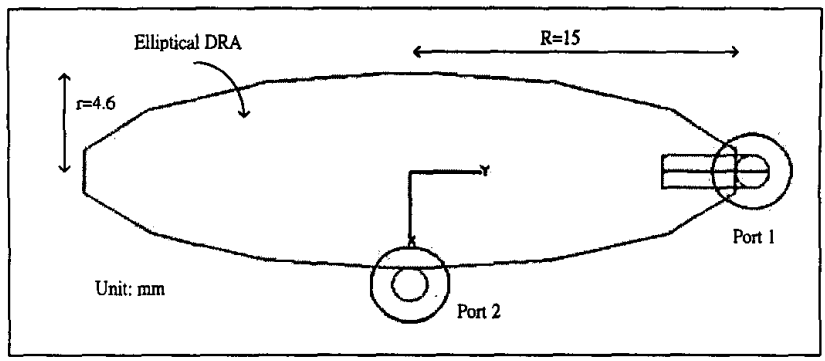

Fig. 1: Top view of the elliptical DRA

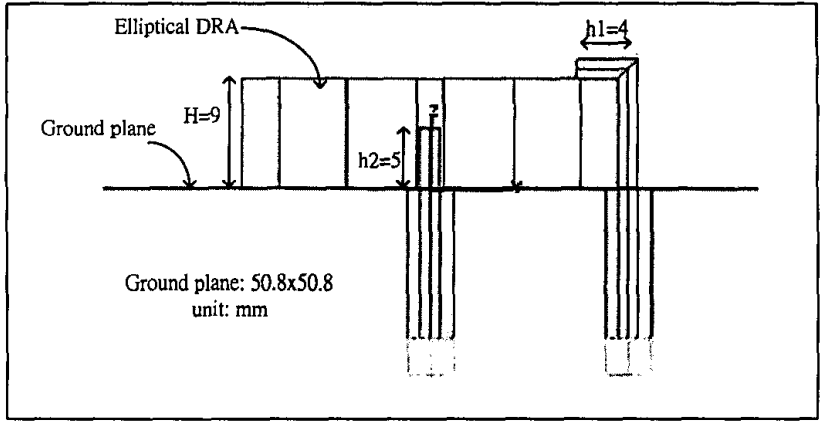

Fig. 2: Side view of the elliptical DRA

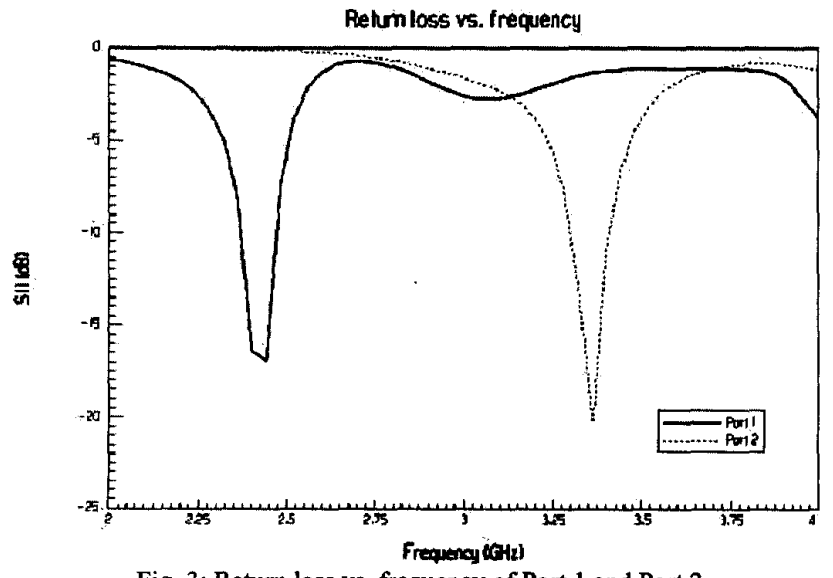

Fig. 3: Return loss vs. frequency of Port 1 and Port 2 


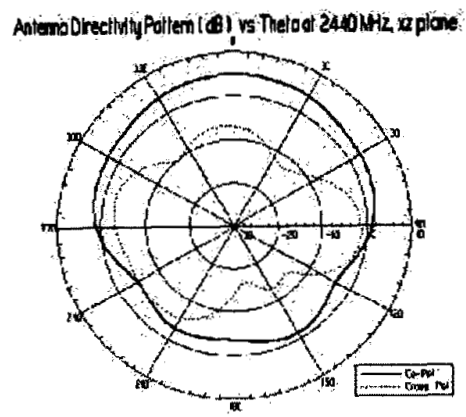

Fig. 4: H-plane pattern of the elliptical $\mathrm{DRA}$ at $2.44 \mathrm{GHz}$

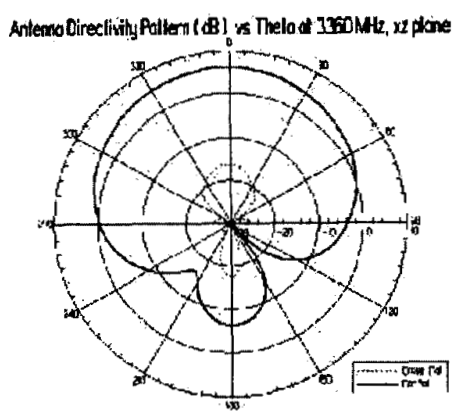

Fig. 6: E-plane pattern of the elliptical DRA at $3.36 \mathrm{GHz}$

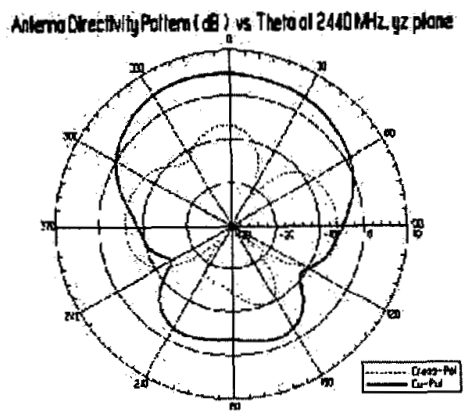

Fig. 5: E-plane pattern of the elliptical DRA at $2.44 \mathrm{GHz}$

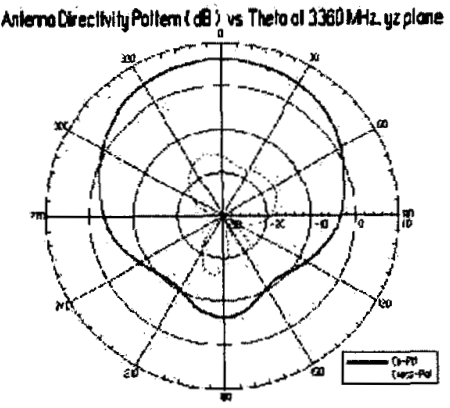

Fig. 7: H-plane pattern of the elliptical DRA at $3.36 \mathrm{GHz}$ 